\title{
ESTRUCTURA Y COMPOSICIÓN FLORÍSTICA DE UN MATORRAL BAJO PLANTACIONES DE PINUS RADIATA D.DON EN CHILE CENTRAL
}

\author{
STRUCTURE AND FLORISTIC COMPOSITION OF A SHRUB UNDER PINUS \\ RADIATA D.DON PLANTATION IN CENTRAL CHILE
}

\author{
Persy Gómez ${ }^{1}$, Steffen Hahn ${ }^{1} \&$ José San Martín² \\ ${ }^{1}$ Jardín Botánico, Universidad de Talca, Casilla 747, Talca, Chile; ${ }^{2}$ Instituto de Biología Vegetal y Biotecnología, \\ Universidad de Talca, Casilla 747, Talca, Chile \\ jbotanico@utalca.cl
}

\section{RESUMEN}

Se analiza la estructura y composición florística de un matorral bajo una plantación de Pinus radiata D.Don en el área costera, Región del Maule ( $35^{\circ} 27^{\prime} 43,3^{\prime}$ 'S; $72^{\circ} 23^{\prime} 56^{\prime}$ 'O), Chile central. Con nueve censos de vegetación se inventarió la flora vascular determinando la riqueza de especies, frecuencia, cobertura-abundancia, valor de importancia junto con origen fitogeográfico y forma de vida. La vegetación se compone de 48 especies distribuidas en 37 nativas, donde 19 son endémicas del país, y 11 introducidas. Dentro de las especies endémicas para el área costera maulina destaca Adesmia bijuga Phil. (Fabaceae). El matorral muestra una organización vertical en estratos donde la mayor cobertura corresponde al estrato arbustivo seguido del herbáceo y el arbóreo. La mayor riqueza con 22 especies se concentra en el estrato herbáceo seguido del arbustivo con 21. Dentro de los componentes florísticos, la más alta frecuencia corresponde a Pinus radiata, Adesmia bijuga Phil. y Escallonia pulverulenta Pers. Los mayores valores de importancia se dan en las especies introducidas Aira caryophyllea L. y Pinus radiata. Las formas de vida representan al conjunto del espectro de Raunkiaer, con dominio de los fanerófitos y hemicriptófitos. Se concluye que la estructura del matorral es variable, con mezcla de especies y una organización compleja dada la presencia de más de un estrato. En un hábitat de extrema artificialización por sustitución de la vegetación original, las especies nativas y dentro de ellas las endémicas muestran capacidad de regeneración y rebrote. Aquí sobresale Adesmia bijuga, que al fructificar sugiere una viabilidad poblacional potencial, la que puede favorecer su conservación in situ bajo el dosel, al menos temporalmente en un hábitat antropogénico, pero con riesgo de amenaza en su supervivencia. Pese a la escasa representatividad de la especie en el área, hasta ahora, y encontrarse fuera de las unidades regionales del SNASPE, la especie aún carece de estatus en su estado de conservación.

Palabras clave: Región del Maule, endémica, nativa, dosel, área costera.

ABSTRACT

The structure and floristic composition of an understory under a Pinus radiata D. Don plantation in the coastal area of the central Chilean Maule Region ( $\left.35^{\circ} 27^{\prime} 43.3^{\prime \prime} \mathrm{S} ; 7^{\circ} 23^{\prime} 56^{\prime \prime} \mathrm{W}\right)$ is documented. Based on nine vegetation samples the vascular flora was inventoried determining species richness, frequency, cover-abundance, and values of importance as well as phytogeographic origin and life forms. The vascular flora comprises 48 taxa with a high representation of native and endemic species (19 each), including Adesmia bijuga Phil. (Fabaceae) as an endemic to the coastal mountain range of Maule Region and only 11 exotic species. The studied shrub community shows a vertical organization in layers of which the herbaceous ground layer shows the highest diversity, whereas Pinus radiata, Adesmia bijuga, and Escallonia pulverulenta Pers. show highest frequency levels. All Raunkiaer life forms are represented, being phanerophytes and hemicryptophytes the dominant life forms. The exotic species Aira caryophyllea L. and Pinus radiata have the highest importance values. We interpret the shrub community structure to be variable and complex due to the artificiallity of the vegetation composition of a planted $P$. radiata stand that replaced the native vegetation. Adesmia bijuga shows that the vital cycle could help to its conservation in situ under the canopy, but in a regime of risk threats its survival. In spite 
Estructura florística de un matorral de Chile central: GómEz, P. ET AL.

of little representativeness in the area the specie is outside the regional units of the SNASPE and is not considered in the Chilean Red Data Book.

KEYworDs: Region of the Maule, endemic, native, canopy, coastal area.

\section{INTRODUCCION}

En la Cordillera de la costa de Chile central, por la ladera occidental y altitudes inferiores a los 100 $\mathrm{m}$, se ha desarrollado un matorral esclerofilo no espinoso (Fuenzalida 1965, Rundell 1981) y en las altas cumbres, bosques caducifolios con especies de Nothofagus (Donoso 1975, Gajardo 1994). Sin embargo, en las últimas décadas por el cambio de uso del suelo, las extensivas plantaciones de Pinus radiata D.Don han reemplazado a la vegetación nativa (Echeverría et al. 2006) con modificación del paisaje original, fragmentación de bosques (Bustamante et al. 2005), pérdida de hábitats, y procesos invasivos de especies desde la matriz circundante hacia las comunidades nativas (Pauchard et al. 2006).

A pesar del impacto negativo de las plantaciones forestales sobre la biodiversidad (Muñoz \& Murúa 1989), Ramírez et al. (1984), para la zona húmeda del país, han reportado una regeneración de especies de la vegetación nativa bajo dosel de Pinus radiata. Por otro lado, y para la zona central, Guerrero \& Bustamante (2007) sugieren que plantaciones de $P$. radiata pueden representar un hábitat potencial para la regeneración y recuperación de algunas especies nativas como Cryptocarya alba (Molina) Looser. Si bien para los bosques nativos maulinos costeros de superficie continua y/o fragmentada, la especie forestal cultivada no presenta una invasión significativa (Bustamante \& Simonetti 2005) para el caso de los matorrales nativos aún es desconocido su impacto.

El escenario anteriormente descrito representa una amenaza para la biodiversidad en especial para especies con reducida distribución y hábitat coincidente con las plantaciones forestales (Sala et al. 2000). Esta situación sugiere un desafío para la conservación, en especial la región maulina reconocida como centro de diversidad de plantas (Villagrán \& Le Quesne 1996, San Martín 2005, Arroyo et al. 2005) y de endemismos con reducidos patrones distribucionales (Smith-Ramírez 2004), por lo tanto es sugerida como área prioritaria para su conservación (Armesto et al.1998, Myers et al. 2000). Precisamente dentro de un matorral costero, y para un sitio, se ha recolectado Adesmia bijuga Phil. con hábito arbustivo y crecimiento semipostrado a erguido (Hahn \& Gómez 2008). La relevancia florística y biogeográfica de la especie radica en el carácter endémico y restringida distribución espacial (Philippi 1884, Reiche 1897, Hahn \& Gómez 2008). En coherencia con el valor biológico de la diversidad florística, en este trabajo se describe la estructura y composición florística de un matorral costero bajo una plantación de $P$. radiata para un sitio donde se desarrolla $A$. bijuga.

Este trabajo pretende contribuir con información sobre la relación de las especies y las formaciones vegetales nativas con las plantaciones forestales, siendo que la mayor parte de los estudios hasta ahora se ha hecho en bosques y matorrales remanentes aún no sustituidos (Rundell 1981, San Martín \& Donoso 1995, San Martín et al. 2001, San Martín 2005, Arroyo et al. 2005). Tal información cobra valor cuando en los planes de conservación in situ se propone restaurar la especie de interés en el contexto de sus otros elementos florísticos acompañantes.

\section{MATERIALES Y METODOS}

Area de estudio

El sitio se ubica en el predio forestal San Pedro, patrimonio de la empresa privada Mininco (35'29'31' S y 72²2’47’ O), comuna de Constitución, provincia de Talca, Región del Maule (Fig. 1). La topografía es montañosa de ondulaciones suaves y elevaciones de hasta 400 $\mathrm{m}$ de altitud. El sustrato geológico es basamento cristalino de rocas metamórficas y material granítico (Mardones 1995) sobre el cual se ha desarrollado un suelo rojo mediterráneo de la serie suelos de Constitución (Pinochet 1991).

El clima es templado mediterráneo con una temperatura media anual de $18{ }^{\circ} \mathrm{C}$ y una precipitación media anual de $709 \mathrm{~mm}$ concentradas en el período invernal (Ulriksen et al. 1979).

La topografía del sitio corresponde a laderas y fondos de quebradas alejadas de la influencia marina, y específicamente el lugar de estudio se sitúa 

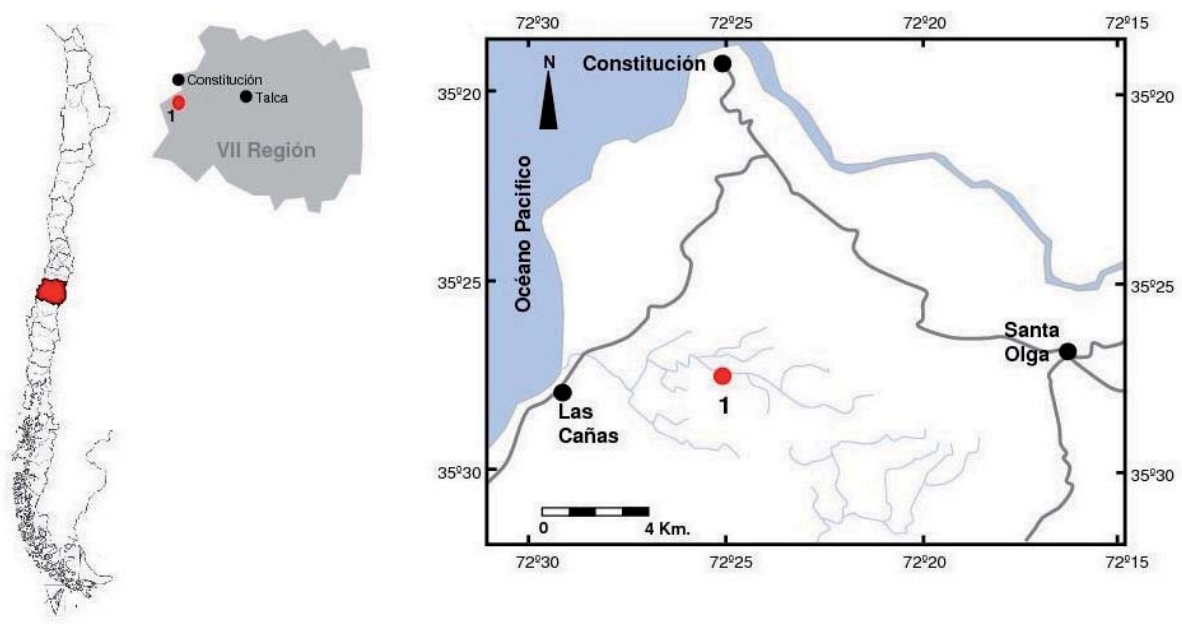

Figura 1. Ubicación del sitio de estudio en la zona costera de la Región del Maule.

FIGURE 1. Location of the study site in the coastal zone of Maule Region.

en una planicie con orientación sur-oeste que, por el borde sur, abruptamente remata en una quebrada. Como consecuencia del cambio de uso del suelo, el paisaje de la vegetación se encuentra alterado y transformado en una combinación de plantación forestal de $P$. radiata y especies nativas. Dentro de este último grupo destacan Nothofagus glauca (Phil.) Krasser con Ageratina glechonophylla (Less.) R.M.King et H.Rob y Sophora macrocarpa $\mathrm{Sm}$. del bosque caducifolio templado (Donoso 1996). Entre las especies del matorral esclerofilo siempreverde están Azara integrifolia Ruiz et Pav., Lithrea caustica (Molina) Hook. et Arn. y Peumus boldus Molina, que pueden alcanzar el crecimiento arbóreo, y arbustos como Podanthus ovatifolius Lag. y Aristeguietia salvia (Colla) R.M.King et H.Rob. El matorral nativo descrito bajo la plantación representa una fase posterior a las faenas de roce con regeneración de especies, las que aparentemente, se ven favorecidas por la moderada intervención del sitio, baja densidad y una corta edad y escasa cobertura de las plantaciones forestales. La recuperación ha sido posible también por la ausencia del género Chusquea que para otros sitios su crecimiento y cobertura han impedido la recolonización de las especies originarias (San Martín \& Donoso 1995). En esta formación de matorral, en el año 2003 se encontró Adesmia bijuga (35²9'31'S; 72²2'47'O). En ese año y luego en el 2006 se recolectó, herborizó y depositó material en el Herbario de la Universidad de Talca
(CHILE, Región del Maule, Prov. Talca, quebrada Fundo San Pedro, 285 y 320 m, 9-XI-2003 y 10-II2006, S. Hahn 2916, P. Gómez \& A. Stoll 3141).

\section{Metodología}

En un sector de una plantación forestal del predio San Pedro se realizó un recorrido e inspección de un área aproximada de 4 ha. Luego, en el interior y bajo individuos de Pinus radiata con alturas superiores a los cinco metros, y crecimiento y cobertura homogénea de los arbustos bajo dosel, se determinó el área mínima según Braun-Blanquet (1979), a partir de $1 \mathrm{~m}^{2}$ de superficie y con inclusión de $P$. radiata se registraron las especies presentes. Luego la superficie se duplicó repetidas veces, registrando sólo las especies nuevas con cada aumento del área, deteniendo el proceso hasta que no se encuentran otras nuevas. Con la información se construye la curva número de especies vs. área y en el punto en que la curva cambia de dirección se proyecta una vertical al eje que representa el área. El punto de intersección indica la menor superficie o área mínima a considerar para la comunidad y caracterización como asociación vegetal sobre la base de información estrictamente florística (Dierschke 1994, Knapp 1984). Respecto de la diversidad del matorral bajo el dosel de la plantación, se trabajó en nueve parcelas, cada una con una superficie de $200 \mathrm{~m}^{2}$, distribuidas al azar y a interdistancia de $70 \mathrm{~m}$, levantando en cada una censos florísticos con registro de las 
Estructura florística de un matorral de Chile central: GómEz, P. ET AL.

especies vasculares por estratos (sin recolección de ejemplares). Para cada especie se determinó su abundancia como porcentaje de cobertura de la parcela (Mueller-Dombois \& Ellenberg 1974, Ramírez et al. 1997). En tablas se recopiló la riqueza de especies, frecuencia, origen geográfico (Gajardo 1983, Matthei 1995) y formas de vida de Raunkiaer (Ellenberg \& Mueller-Dombois 1966). Con las frecuencias y coberturas relativas de cada especie en todos los censos se calculó el valor de importancia (Wikum \& Shanholtzer 1978). La frecuencia relativa se obtiene de la suma de las frecuencias absolutas (censos donde las especies están presentes) de todas las especies llevando el total a 100 y determinando el porcentaje del total que corresponde a cada especie. El cálculo para la cobertura relativa sigue el mismo procedimiento. La suma de la frecuencia relativa con la cobertura relativa de cada especie entrega un valor que en este caso como máximo no supera los 200. El valor de importancia expresa la magnitud y rol de participación de un taxón en la comunidad, siendo útil sólo para el análisis de la tabla vegetacional (San Martín et al 1992). La nomenclatura de los taxa sigue Rodríguez et al. (1983) y Zuloaga et al. (2008).

\section{RESULTADOS}

RiQuEZA, ORIGEN GEOGRÁFICO DE LAS ESPECIES Y ESPECTRO BIOLÓGICO DE LA VEGETACIÓN DEL SITIO Para una superficie muestreada de $1.800 \mathrm{~m}^{2}$ la riqueza florística del matorral con $P$. radiata incluye 48 especies de plantas vasculares (Tabla I y Anexo I) distribuidas en 27 familias y 46 géneros.

TABLA I. Censos ordenados según estratos y valores porcentuales de cobertura.

TABLE I. Vegetation samples ordered by layers and percentage values of coverage.

\begin{tabular}{|c|c|c|c|c|c|c|c|c|c|c|c|}
\hline CENSO & 1 & 2 & 3 & 4 & 5 & 6 & 7 & 8 & 9 & $\% \mathrm{~F}$ & $\% \mathrm{C}$ \\
\hline Altitud (m) & & 322 & 322 & 318 & 320 & 320 & 315 & 320 & 321 & & \\
\hline Orientación & & $\mathrm{NE}$ & $\mathrm{NE}$ & SE & SE & SE & $\mathrm{NE}$ & SE & $\mathrm{NE}$ & & \\
\hline Inclinación \% & 2 & 2 & 2 & 2 & 2 & 2 & 5 & 2 & 2 & & \\
\hline $\mathrm{N}^{\circ}$ especies & 7 & 7 & 10 & 10 & 12 & 11 & 12 & 9 & 10 & & \\
\hline \multicolumn{12}{|l|}{ ESTRATO ARBÓREO } \\
\hline Pinus radiata & 15 & & 40 & & 25 & 10 & & 10 & 30 & 66,7 & 14,5 \\
\hline \multicolumn{12}{|l|}{ ESTRATO ARBUSTIVO } \\
\hline Baccharis concava & & 10 & & & & 20 & & 15 & & 33,3 & 5,0 \\
\hline Escallonia pulverulenta & & 20 & & & 5 & 3 & & 10 & & 44,4 & 4,2 \\
\hline Adesmia bijuga & 13 & & 10 & & 5 & & & 10 & & 44,4 & 4,2 \\
\hline Adesmia elegans & & & 10 & & 5 & 20 & & & & 33,3 & 3,9 \\
\hline Peumus boldus & 15 & 20 & & & & & & & & 22,2 & 3,9 \\
\hline Sophora macrocarpa & & & & 10 & 5 & 20 & & & & 33,3 & 3,9 \\
\hline Ugni molinae & & 10 & & & & & & 25 & & 22,2 & 3,9 \\
\hline Lithrea caustica & 15 & & & & 5 & & & 10 & & 33,3 & 3,3 \\
\hline Maytenus boaria & & & & & & & 30 & & & 11,1 & 3,3 \\
\hline Ribes punctatum & 13 & & & & 5 & 10 & & & & 33,3 & 3,1 \\
\hline Aristotelia chilensis & & & 10 & & & 5 & & 10 & & 33,3 & 2,8 \\
\hline Luma apiculata & & 20 & & & & & & & & 11,1 & 2,2 \\
\hline Cryptocarya alba & 15 & & & & & 2 & & & & 22,2 & 1,9 \\
\hline Persea lingue & 15 & & & & & 2 & & & & 22,2 & 1,9 \\
\hline Calceolaria integrifolia & & & 5 & & & & 5 & & & 22,2 & 1,1 \\
\hline Rosa rubiginosa & & & 5 & & 5 & & & & & 22,2 & 1,1 \\
\hline Teucrium bicolor & & & & 10 & & & & & & 11,1 & 1,1 \\
\hline Lomatia hirsuta & & & & & & & & 5 & & 11,1 & 0,6 \\
\hline Nothofagus glauca & & & & & & & 5 & & & 11,1 & 0,6 \\
\hline
\end{tabular}


Gayana Bot. 66(2), 2009

\begin{tabular}{|c|c|c|c|c|c|c|c|c|c|c|c|}
\hline CENSO & 1 & 2 & 3 & 4 & 5 & 6 & 7 & 8 & 9 & $\% \mathrm{~F}$ & $\% \mathrm{C}$ \\
\hline Podanthus ovatifolius & & & & & & 5 & & & & 11,1 & 0,6 \\
\hline Kageneckia oblonga & & & & & & 3 & & & & 11,1 & 0,3 \\
\hline \multicolumn{12}{|l|}{ ESTRATO HERBÁCEO } \\
\hline Aira caryophyllea & & & & 40 & 30 & & & & 20 & 33,3 & 10,0 \\
\hline Nassella chilensis & & & & 10 & & & 20 & & 5 & 33,3 & 3,9 \\
\hline Alstroemeria revoluta & & & & & & & & & 20 & 11,1 & 2,2 \\
\hline Acaena argentea & & 10 & & & & & & & 5 & 22,3 & 1,7 \\
\hline Eryngium paniculatum & & & & & & & 10 & & & 11,1 & 1,1 \\
\hline Hypochaeris radicata & & & & 5 & 5 & & & & & 22,2 & 1,1 \\
\hline Lobelia excelsa & & 10 & & & & & & & & 11,1 & 1,1 \\
\hline Stachys macraei & & & & & & & 5 & & 5 & 22,2 & 1,1 \\
\hline Salpiglossis sinuata & & & & & 4 & & 5 & & & 22,2 & 1,0 \\
\hline Rumex acetosella & & & & 5 & & & & & 1 & 22,2 & 0,7 \\
\hline Carduus pycnocephalus & & & & & & & 5 & & & 11,1 & 0,6 \\
\hline Cynara cardunculus & & & 5 & & & & & & & 11,1 & 0,6 \\
\hline Daucus carota & & & & 5 & & & & & & 11,1 & 0,6 \\
\hline Gnaphalium cheiranthifolium & & & & 5 & & & & & & 11,1 & 0,6 \\
\hline Lactuca serriola & & & 5 & & & & & & & 11,1 & 0,6 \\
\hline Leontodon saxatilis & & & 5 & & & & & & & 11,1 & 0,6 \\
\hline Mulinum spinosum & & & & & & & & & 5 & 11,1 & 0,6 \\
\hline Pasithea caerulea & & & & & & & & & 5 & 11,1 & 0,6 \\
\hline Plantago lanceolada & & & 5 & & & & & & & 11,1 & 0,6 \\
\hline Alstroemeria ligtu & & & & & & & 4 & & & 11,1 & 0,4 \\
\hline Sisyrinchium chilense & & & & & & & & & 4 & 11,1 & 0,4 \\
\hline Adiantum chilense & & & & & & & 1 & & & 11,1 & 0,1 \\
\hline \multicolumn{12}{|l|}{ Trepadoras } \\
\hline Muehlenbeckia hastulata & & & & 5 & & & 5 & & & 22,2 & 1,1 \\
\hline Bomarea salsilla & & & & & & & 5 & & & 11,1 & 0,6 \\
\hline Lathyrus magellanicus & & & & 5 & & & & & & 11,1 & 0,6 \\
\hline Galium cotinoides & & & & & 1 & & & & & 11,1 & 0,1 \\
\hline
\end{tabular}

ANEXo 1. Nombres científicos, familias, formas de vida (Fv), origen fitogeográfico (O), y valor de importancia (VI) de las especies del sitio de estudio. $\mathrm{Fa}=$ fanerófitos; $\mathrm{Ca}=$ caméfitos; $\mathrm{Hc}=$ hemicriptófitos; $\mathrm{Cr}=$ criptófitos y Te= terófitos.

AnNEX 1. Scientific names, families, life forms (Fv), phytogeographic origin (O) and value of importance (VI) of the study site's flora. $\mathrm{Fa}=$ phanerophytes; $\mathrm{Ca}=$ chamaephytes; $\mathrm{Hc}=$; hemicryptophytes; $\mathrm{Cr}=$ Cryptophytes and $\mathrm{Te}=$ therophytes.

\begin{tabular}{llllc}
\hline Nombre científico & Familia & Fv & O & VI \\
\hline Pinus radiata D.Don & Pinaceae & Fa & I & 21,5 \\
Aira caryophyllea L. & Poaceae & Te & I & 13,5 \\
Adesmia bijuga Phil. & Fabaceae & Ca & E & 8,9 \\
Escallonia pulverulenta Pers. & Escalloniaceae & Fa & E & 8,9 \\
Baccharis concava Pers. & Asteraceae & Fa & N & 8,5 \\
Adesmia elegans Clos & Fabaceae & Ca & E & 7,4 \\
Nassella chilensis E.Desv. & Poaceae & $\mathrm{Hc}$ & $\mathrm{E}$ & 7,4 \\
Sophora macrocarpa Sm. & Fabaceae & $\mathrm{Fa}$ & $\mathrm{E}$ & 7,4 \\
Lithrea caustica Hook. et Arn. & Anacardiaceae & $\mathrm{Fa}$ & $\mathrm{E}$ & 6,8 \\
Ribes punctatum Ruiz et Pav. & Grossulariaceae & $\mathrm{Fa}$ & $\mathrm{N}$ & 6,6 \\
Aristotelia chilensis Stuntz & Elaeocarpaceae & $\mathrm{Fa}$ & $\mathrm{N}$ & 6,3 \\
Peumus boldus Molina & Monimiaceae & $\mathrm{Fa}$ & $\mathrm{E}$ & 6,2 \\
\hline
\end{tabular}


Estructura florística de un matorral de Chile central: GómEz, P. ET AL.

\begin{tabular}{|c|c|c|c|c|}
\hline Nombre científico & Familia & $\mathrm{FV}$ & $\mathrm{O}$ & $\mathrm{VI}$ \\
\hline Ugni molinae Turcz. & Myrtaceae & $\mathrm{Fa}$ & $\mathrm{N}$ & 6,2 \\
\hline Maytenus boaria Molina & Celastraceae & $\mathrm{Fa}$ & $\mathrm{N}$ & 4,5 \\
\hline Cryptocarya alba (Molina) Looser & Lauraceae & $\mathrm{Fa}$ & $\mathrm{E}$ & 4,2 \\
\hline Persea lingue (Miers ex Bertero) Nees & Lauraceae & $\mathrm{Fa}$ & $\mathrm{N}$ & 4,2 \\
\hline Acaena argentea Ruiz et Pav. & Rosaceae & $\mathrm{Ca}$ & $\mathrm{N}$ & 4,0 \\
\hline Alstroemeria revoluta Ruiz et Pav. & Alstroemeriaceae & $\mathrm{Cr}$ & $\mathrm{E}$ & 3,4 \\
\hline Calceolaria integrifolia $\mathrm{L}$. & Calceolariaceae & $\mathrm{Ca}$ & $\mathrm{E}$ & 3,4 \\
\hline Hypochaeris radicata $\mathrm{L}$. & Asteraceae & $\mathrm{Hc}$ & $\mathrm{I}$ & 3,4 \\
\hline Luma apiculata (DC.) Burret & Myrtaceae & $\mathrm{Fa}$ & $\mathrm{N}$ & 3,4 \\
\hline Rosa rubiginosa $\mathrm{L}$. & Rosaceae & $\mathrm{Fa}$ & $\mathrm{I}$ & 3,4 \\
\hline Stachys macraei Benth. & Lamiaceae & $\mathrm{Ca}$ & $\mathrm{E}$ & 3,4 \\
\hline Salpiglossis sinuata Ruiz et Pav. & Solanaceae & $\mathrm{Hc}$ & $\mathrm{E}$ & 3,3 \\
\hline Rumex acetosella $\mathrm{L}$. & Polygonaceae & $\mathrm{Hc}$ & $\mathrm{I}$ & 3,0 \\
\hline Eryngium paniculatum Cav. et Domb. ex Delar. & Apiaceae & $\mathrm{Hc}$ & $\mathrm{N}$ & 2,3 \\
\hline Lobelia excelsa Bonpl. & Campanulaceae & $\mathrm{Fa}$ & $\mathrm{E}$ & 2,3 \\
\hline Muehlenbeckia hastulata I.M.Johnst. & Polygonaceae & $\mathrm{Fa}$ & $\mathrm{N}$ & 2,3 \\
\hline Teucrium bicolor Sm. & Lamiaceae & $\mathrm{Fa}$ & $\mathrm{N}$ & 2,3 \\
\hline Bomarea salsilla Mirb. & Alstroemeriaceae & $\mathrm{Cr}$ & $\mathrm{E}$ & 1,8 \\
\hline Carduus pycnocephalus L. & Asteraceae & $\mathrm{Te}$ & $\mathrm{I}$ & 1,8 \\
\hline Cynara cardunculus L. & Asteraceae & $\mathrm{Te}$ & I & 1,8 \\
\hline Daucus carota L. & Apiaceae & $\mathrm{Hc}$ & I & 1,8 \\
\hline Gnaphalium cheiranthifolium Bertero ex DC. & Asteraceae & $\mathrm{Hc}$ & $\mathrm{N}$ & 1,8 \\
\hline Lactuca serriola L. & Asteraceae & $\mathrm{Te}$ & I & 1,8 \\
\hline Lathyrus magellanicus Lam. & Fabaceae & $\mathrm{Te}$ & $\mathrm{N}$ & 1,8 \\
\hline Leontodon taraxacoides (Willd.) Merat & Asteraceae & $\mathrm{Hc}$ & I & 1,8 \\
\hline Lomatia hirsuta (Lam.) Diels & Proteaceae & $\mathrm{Fa}$ & $\mathrm{N}$ & 1,8 \\
\hline Mulinum spinosum (Cav.) Pers. & Apiaceae & $\mathrm{Hc}$ & $\mathrm{N}$ & 1,8 \\
\hline Nothofagus glauca (Phil.) Krasser & Nothofagaceae & $\mathrm{Fa}$ & $\mathrm{E}$ & 1,8 \\
\hline Pasithea caerulea (Ruiz et Pav.) D.Don & Hemerocallidaceae & $\mathrm{Cr}$ & $\mathrm{E}$ & 1,8 \\
\hline Podanthus ovatifolius Lag. & Asteraceae & $\mathrm{Fa}$ & $\mathrm{N}$ & 1,8 \\
\hline Plantago lanceolata $\mathrm{L}$. & Plantaginaceae & $\mathrm{Hc}$ & $\mathrm{I}$ & 1,8 \\
\hline Alstroemeria ligtu $\mathrm{L}$. & Alstroemeriaceae & $\mathrm{Cr}$ & $\mathrm{E}$ & 1,6 \\
\hline Kageneckia oblonga Ruiz et Pav. & Rosaceae & $\mathrm{Fa}$ & $\mathrm{E}$ & 1,5 \\
\hline Adiantum chilense Kaulf. & Adiantaceae & $\mathrm{Hc}$ & $\mathrm{N}$ & 1,3 \\
\hline Galium cotinoides Cham. et Schltdl. & Rubiaceae & $\mathrm{Ca}$ & $\mathrm{E}$ & 1,3 \\
\hline Sisyrinchium chilense Hook. & Iridaceae & $\mathrm{Cr}$ & $\mathrm{N}$ & 1,3 \\
\hline
\end{tabular}

En el origen geográfico mayoritariamente está representado el grupo de las nativas con 37 especies. En este conjunto se incluyen 19 endémicas (39,6\% del total), sobresaliendo Adesmia elegans Clos y $A$. biguja como restringida al área costera de la región maulina (Reiche 1897, Burkart 1967), mientras que las otras 17 especies se encuentran en otras regiones del país (Gajardo 1983). Como nativas no endémicas se encuentran otras 18 especies. Las especies introducidas corresponden al 22,92\% (10 especies), donde se incluye $P$. radiata, cultivado, y las demás son malezas en praderas y cultivos (Matthei 1995) (Tabla III y Anexo I).

Todas las formas de vida sensu Raunkiaer están representadas. Mientras que dominan los fanerófitos y hemicriptófitos, los caméfitos, criptófitos y terófitos están pobremente representados (Fig. 2 y Tabla III). Aquí los fanerófitos incluyen 21 especies de las cuales ocho son endémicas.

$\mathrm{Al}$ combinar el origen geográfico con las formas de vida se observa que fanerófitas y hemicriptófitas concentran la mayor cantidad de especies nativas endémicas y no endémicas con 11 y 14 elementos respectivamente. Caméfitas y criptófitas no se dan en el grupo de las introducidas como también las terófitas en las especies endémicas (Tabla III). 
Gayana Bot. 66(2), 2009

TABLA II. Riqueza de especies y valores de cobertura absolutos y porcentuales para los diferentes estratos de la vegetación sitio de estudio.

TABLE II. Species richness and absolute and percentage values of cover for the layers of the study site.

\begin{tabular}{lccc}
\hline Estrato & $\mathrm{N}^{\circ}$ especies & Cobertura & \% Cobertura \\
\hline Arbóreo & 1 & 130 & 14,5 \\
Arbustivo & 21 & 476 & 53,2 \\
Herbáceo & 22 & 269 & 30,0 \\
Trepador & 4 & 21 & 2,3 \\
Total & 48 & 896 & 100,0 \\
\hline
\end{tabular}

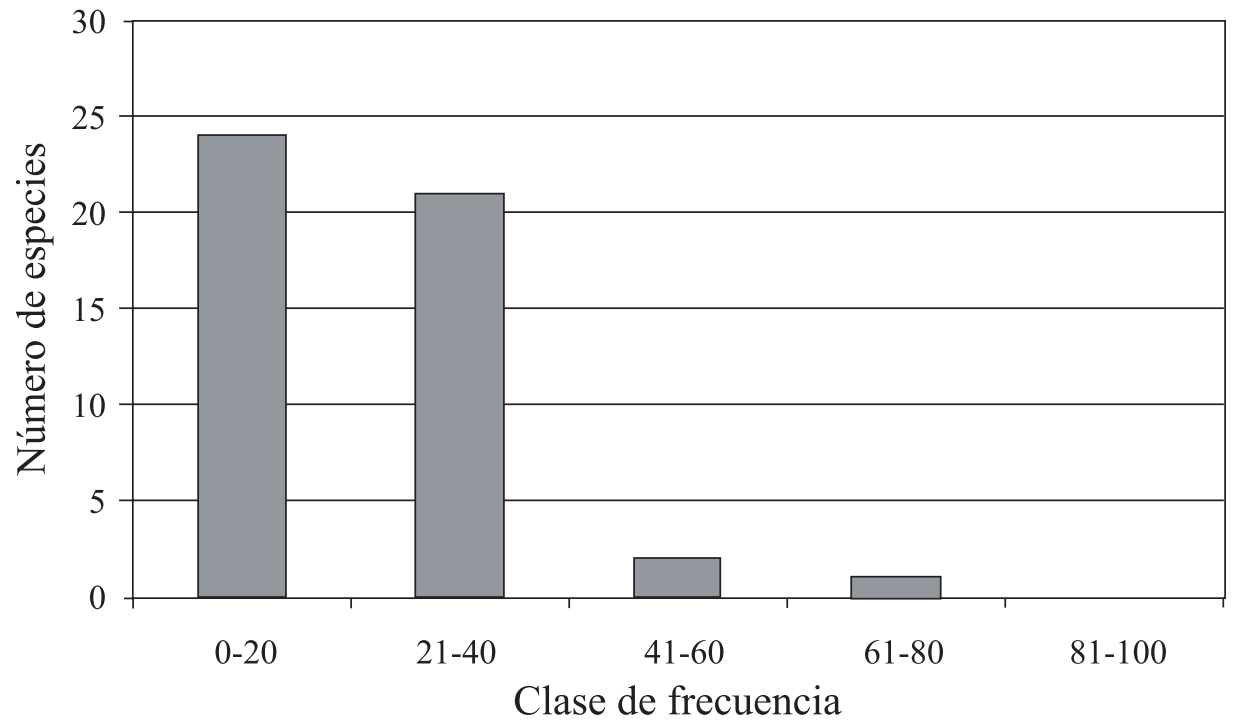

Figura 2. Histograma de las clases de frecuencias para la flora en un matorral bajo el dosel de Pinus radiata de la zona costera de la Región del Maule.

Figure 2. Frequency classes histogram of a shrub community under the canopy of Pinus radiata of the coastal zone of Maule Region.

ESTRATIFICACIÓN; FRECUENCIA Y COBERTURAABUNDANCIA

A causa de las diversas formas de crecimiento, los elementos florísticos tienden a una organización vertical en tres estratos definidos: herbáceo, arbustivo y arbóreo (Tablas I y II). Pinus radiata como especie única define el estrato arbóreo. Contrariamente las 21 especies arbustivas definen la composición del matorral. Como arbustos típicos están A. bijuga, A. elegans Clos, Baccharis concava (Ruiz et Pav.) Pers., Calceolaria integrifolia L., Podanthus ovatifolius Lag., Ribes punctatum Ruiz et Pav., Rosa rubiginosa L., Sophora macrocarpa Sm., Teucrium bicolor Sm. y Ugni molinae Turcz.
(Tabla I). A este estrato se agregan otras especies cuyo normal desarrollo es arbóreo, pero que aquí participan en el nivel arbustivo como Aristotelia chilensis Stuntz, Cryptocarya alba Ruiz et Pav., Escallonia pulverulenta Pers., Kageneckia oblonga Ruiz et Pav., Lithrea caustica, Lomatia hirsuta (Lam.) Diels, Luma apiculata (DC.) Burret, Maytenus boaria Molina, Nothofagus glauca, Persea lingue (Miers ex Bertero) Nees y Peumus boldus (Tabla I). Finalmente el estrato herbáceo con 22 especies representa la mayor riqueza con elementos nativos e introducidos. Como especies nativas, entre otras, están Acaena argentea Ruiz et Pav., Adiantum chilense Kaulf., Alstroemeria 
Estructura florística de un matorral de Chile central: GómEz, P. ET AL.

TABLA III. Valores absolutos de las formas de vida según origen geográfico de la flora del sitio de estudio.

TABLE III. Absolute values of the life forms according to geographic origin of the study site's flora.

\begin{tabular}{lcccc}
\hline & \multicolumn{3}{c}{ Origen geográfico } & \\
\cline { 2 - 3 } Formas de vida & \multicolumn{2}{c}{ Nativas } & Introducidas & Total \\
\cline { 2 - 3 } & Endémicas & No Endémicas & & \\
\hline Fanerófitas & 8 & 11 & 2 & 21 \\
Caméfitas & 5 & 1 & 0 & 6 \\
Hemicriptófitas & 3 & 3 & 5 & 11 \\
Criptófitas & 4 & 1 & 0 & 5 \\
Terófitas & 0 & 1 & 4 & 5 \\
Total & 20 & 17 & 11 & 48 \\
$\%$ & 41,66 & 35,42 & 22,92 & 100 \\
\hline
\end{tabular}

ligtu L., A. revoluta Ruiz et Pav., Eryngium paniculatum Cav., Lobelia excelsa, etc. Como representantes introducidos y con carácter de malezas destacan Aira caryophyllea L., Carduus pycnocephalus L. Cynara cardunculus L., Daucus carota L., etc. (Tabla I).

Como resultado de las frecuencias se observan valores dispares entre las especies con un máximo de $66,7 \%$ y un mínimo de $11,1 \%$. Las mayores frecuencias (superiores a 33,3\%), se concentran sólo en 11 especies $\mathrm{y}$, contrariamente, valores inferiores a $22,2 \%$ concentran la mayor cantidad (37 sp.). Como era esperable la mayor frecuencia de $66,7 \%$ corresponde a $P$. radiata de la plantación forestal. Con un $44,4 \%$ le se siguen $E$. pulverulenta y $A$. bijuga del estrato arbustivo, con un $33,3 \%$ siguen $B$. concava, A. elegans, $S$. macrocarpa, $L$. caustica, $R$. punctatum y A. chilensis, y entre las hierbas A. caryophyllea y Nassella chilensis E. Desv. Las restantes especies de arbustos, hierbas y trepadoras presentan frecuencias menores que varían entre 22,2 y $11,1 \%$ (Tabla I).

$\mathrm{Al}$ ordenar las especies de acuerdo a los valores porcentuales de frecuencia en un histograma de Raunkiaer (Mateucci \& Colma 1982), se observa que la mayor cantidad se concentra en las clases 0 20 y $21-40 \%$ con ausencia de representación para $80-100 \%$ (Fig. 2).

La cobertura de la vegetación en relación con la superficie muestreada es baja por cuanto alcanza sólo a un 49,8\% $\left(896 \mathrm{~m}^{2}\right)$, y tanto para los estratos como para las especies no es uniforme. Al comparar los estratos la mayor cobertura es para los arbustos con un 53,2\% $\left(476 \mathrm{~m}^{2}\right)$ seguidos del estrato herbáceo con un 30,0\% $\left(269 \mathrm{~m}^{2}\right)$. Le sigue el estrato arbóreo con sólo un 14,5 \% (130 m²). La menor cobertura se da en las trepadoras con sólo un 2,3\% (21 $\left.\mathrm{m}^{2}\right)$ (Tabla II). Tal situación confirma el estado juvenil de las plantaciones de $P$. radiata dejando aún espacio disponible para la entrada de luz, y con ello ha permitido una recuperación de los estratos herbáceos y arbustivos. Sin embargo, al analizar la cobertura por especie y por estrato la situación es diferente. Para el nivel arbóreo la cobertura absoluta mayor es para $P$. radiata con $130 \mathrm{~m}^{2}$. Dentro del estrato arbustivo la cobertura mayor la presenta Baccharis concava con $45 \mathrm{~m}^{2}$ seguida de Escallonia pulverulenta y Adesmia bijuga con $38 \mathrm{~m}^{2}$ cada una. Luego con $35 \mathrm{~m}^{2}$ están Adesmia elegans, Peumus boldus, Sophora macrocarpa y Ugni molinae Turcz. A nivel herbáceo, Aira caryophyllea alcanza una cobertura de $90 \mathrm{~m}^{2}$ seguida de Nassella chilensis E.Desv. con $35 \mathrm{~m}^{2}$. Finalmente entre las trepadoras destaca Muehlenbeckia hastulata I.M.Johnst. con sólo $10 \mathrm{~m}^{2}$ (Tabla I).

VALOR DE IMPORTANCIA DE LAS ESPECIES

Finalmente los valores de importancia son bajos a causa del reducido aporte en cobertura y frecuencia de las especies. Estos valores oscilan entre 21,5 como el más alto y 1,3 el menor. Del conjunto florístico del sitio de estudio, sólo Pinus radiata y Aira caryophyllea presentan valores de importancia más altos, con 21,5 y 13,5 respectivamente. Luego entre 8,9 y 6,2 se incluyen 11 especies. La mayor cantidad de elementos florísticos (35 spp.) presentan valores entre 8,9 y 1,3. Adesmia bijuga junto a Escallonia pulverulenta presentan 8,9 (Anexo I). 


\section{DISCUSION}

Bajo el dosel y en la etapa inicial de las plantaciones de Pinus radiata costeras maulinas y al igual que otras del sur del país (Ramírez et al. 1984) se desarrolla un matorral con especies originales de sitio, así como otras de origen extranjero que en los cultivos agrícolas se comportan como malezas. Entre las especies leñosas con una alta capacidad regenerativa bajo dosel de plantaciones y comunes a la zona central y valdiviana están las nativas Aristotelia chilensis, Baccharis concava, Maytenus boaria, Persea lingue y Ugni molinae y, finalmente, la maleza herbácea Hypochaeris radicata. Una diferencia observada en el matorral de San Pedro respecto al descrito por Ramírez et al. (1984) es la no formulación a una unidad fitosociológica o asociación, ya que ésta se basa en tablas vegetacionales y no en un grupo de censos particulares. Los censos provienen de inventarios en una comunidad con composición, fisonomía y hábitat uniforme (Izco \& Del Arco 2003). Para el sitio de estudio no es el caso, debido a que se trata de un ambiente alterado por el hombre $\mathrm{y}$ vegetacionalmente transformado, donde además se ha establecido una especie introducida.

El matorral representa una sustitución de la vegetación original y específicamente del bosque maulino costero con Nothofagus glauca. La composición florística confirma lo señalado por Donoso (1993) y Bustamante \& Simonetti (2005) en cuanto a que las especies con una mayor capacidad de regeneración vegetativa son elementos del bosque esclerofilo, que en algunos casos pueden desarrollar lignotúberes (Montenegro et al. 1983) como Escallonia pulverulenta, Peumus boldus, Lithrea caustica, Luma apiculata, Cryptocarya alba, Kageneckia oblonga y, de ambientes más húmedos o mésicos, Lomatia hirsuta y Persea lingue. En menor proporción lo hacen especies propias del bosque original como Nothofagus glauca y Sophora macrocarpa. Esta situación contribuye a aumentar la diversidad florística absoluta como lo demuestran Amigo et al. (2000) y Litton \& Santelices (1996) para bosques secundarios de $N$. glauca. La intervención y perturbación del bosque maulino costero se manifiesta, además, con la fragmentación y reducción de la cobertura original, y en oposición al aumento de la superficie de plantaciones forestales, como lo demuestran y analizan Bustamante \& Simonetti
(2005) y Echeverría et al. (2006). El matorral es de composición florística mixta, con presencia de especies de hábitats mésicos y elementos del bosque esclerofilo, donde dentro de este último grupo un elemento interesante es la presencia de Cryptocarya alba. Tal hallazgo valida la propuesta de Guerrero \& Bustamante (2007) en el sentido de que es una especie que sin dificultades aparentes puede regenerar en las plantaciones.

Para el sitio la regeneración de las especies nativas es bajo un régimen de artificialización con las condiciones señaladas por Sukopp (1969), es decir, una acentuada influencia antrópica con destrucción de la comunidad original. La influencia e impacto del hombre se clasifica según su grado en clases de hemerobia y de acuerdo a la escala de esta metodología, el matorral se posiciona en una degradación extrema correspondiéndole el grado de metahemerobia (Stoll 2007, 2008). A causa de la joven plantación forestal, la cobertura del dosel es baja quedando una oferta de claros en que sólo las especies nativas, con capacidad de rebrote, inician una recolonización del espacio representando así un indicador ecológico del régimen de perturbación antrópica (Niemi \& McDonald 2004). Los elementos más favorecidos son también los introducidos, donde la apertura y sustitución del dosel de la vegetación arbórea nativa original genera un espacio aprovechado por especies oportunistas como Aira caryophyllea. Precisamente, en sitios abiertos y/o con sustitución aumenta la disponibilidad de luz y temperatura ya que en la noche y para un sitio abierto la temperatura baja más que dentro de un bosque cerrado, siendo ventajoso para las especies herbáceas (Kimmins 1987). La excepción es para Pinus radiata que es intencionalmente plantado en suelos abiertos y despejados de vegetación nativa. Observaciones de terreno dan cuenta que en plantaciones con cobertura cerrada de copa no se observa una regeneración bajo dosel, excepto posterior a una quema de los desechos postcosechas. Por otro lado, en los fragmentos de bosque nativo no existe certeza si la regeneración de $P$. radiata en el interior es consecuencia de su capacidad invasiva como era esperable en el sentido de Bustamante \& Simonetti (2005). Aparentemente esta limitación de la especie se relacionaría con su condición de intolerancia a la sombra (Richardson 1998). Una situación similar se podría esperar para la flora del sitio de estudio en cuanto a la capacidad de 
tolerancia a baja luminosidad a escala temporal y al aumento de la cobertura del dosel con la edad de la plantación.

De acuerdo con el histograma de clases de frecuencias de Raunkiaer (Fig. 2), el matorral carece aún de una definida organización estructural como unidad comunitaria con un definido patrón estructural. Esto se explica por el alto número de especies con baja frecuencia agrupadas entre las clases $0-20$ y $21-40 \%$ respecto a otras de valores más altos (40-60\%) y que con baja diversidad pueden dominar la organización. La dominancia de estas especies es aparentemente por el grado de artificialización del sitio, y responde a una dinámica de la vegetación cuya dirección sucesional progresiva $\mathrm{y} / \mathrm{o}$ regresiva dependería de las modificaciones de la variable física de la luminosidad por aumento de la cobertura de la plantación (Kimmins 1987).

La dominancia de fanerófitos y hemicriptófitos (Tabla III) indica un fitoclima templado (Cain 1950), lo cual es coincidente con el macroclima costero maulino (Ulriksen et al. 1979) y demuestra que la adecuación morfológica de las plantas a las condiciones ambientales coincide con lo descrito para un clima templado de latitudes medias del hemisferio norte y sur (Cain 1950) donde el ajuste es independiente de la posición sistemática y la amplitud de la distribución ecológica de las especies. La presencia de hemicriptófitos en rosetas como Hypochaeris radicata y Leontodon saxatilis y su capacidad de regenerar a partir de la yema, contribuiría a resistir el pisoteo y que al colonizar sitios intervenidos por el hombre son también indicadores del proceso de antropización. Precisamente, Grime (1989) caracteriza a las especies que colonizan sitios alterados por el hombre como ruderales y es el caso de los elementos que crecen en roseta como los mencionados anteriormente. Frente a otras especies herbáceas este morfotipo es una ventaja para colonizar áreas diferentes a las praderas aunque en esta última formación vegetal son también más frecuentes (Hauenstein et al. 1988, Ramírez et al. 1997, San Martín et al. 2001). El bajo número de terófitos y caméfitos era esperable dado que en el sotobosque tanto de bosques nativos como en los de origen artificial no son frecuentes (Ramírez 1988).

La estructura del matorral es compleja. Ello se explica por las diferentes formas de crecimiento de las especies que verticalmente se ordenan en más de un estrato respecto a la plantación forestal que es monoestratificada o simple. Favorecidos por la corta edad y baja cobertura del dosel de Pinus radiata, un grupo de especies leñosas ha rebrotado ocupando diferentes estratos. Tal situación sugiere que la plantación forestal se estableció previa sustitución de la vegetación original. Su persistencia dependerá de la tolerancia a la sombra, situación que, prácticamente, en la interacción especies nativas vs. plantación forestal no ha sido evaluada. El origen de las especies nativas responde a una estrategia de regeneración vegetativa con diásporas disponibles en el sitio permitido por una débil cobertura de la plantación (Fuentes 1989). Contrariamente, en semillas se ha observado un efecto alelopático de la hojarasca de Pinus radiata (Guerrero \& Bustamante 2007). Los resultados muestran la necesidad de ampliar los estudios en otros matorrales bajo plantación. Es importante monitorear la capacidad de tolerancia de las especies nativas en relación con el efecto de la altura, edad de crecimiento y cobertura de copas de Pinus radiata.

En el matorral bajo el dosel hasta ahora los ejemplares de Adesmia bijuga muestran un buen desarrollo, sugiriendo un reclutamiento por regeneración natural. Sin embargo, a causa del desconocimiento de la ecología de esta especie así como de otras especies nativas no es posible predecir las posibilidades de sobrevivencia y su respuesta a la sombra de la plantación. Fisonómicamente la especie es siempreverde con alturas de crecimiento de 0,15 a $2 \mathrm{~m}$, hojas verde oscuras brillantes y aromáticas al frotarlas. Por frecuentes monitoreos se encontró que cumple su ciclo vital con floración en el período de primavera-verano. La fructificación es entre diciembre y enero y la dispersión de los frutos y semillas, por gravedad, en febrero. Aunque no se disponen de antecedentes para otros sitios, aquí la especie muestra depredación de semillas previa a su dispersión, por el insecto Litraeus poverus, Bruchidae, desconociéndose la viabilidad y valores de germinación de las semillas.

Si bien en el sitio, la disminución de la actividad zooantrópica posplantación ha favorecido su recuperación, a largo plazo no es predecible su persistencia temporal. Los riegos de amenazas derivarán por el cierre de copas de la plantación con disminución de la disponibilidad de luz y más 
tarde las intervenciones de roce y faenas de la cosecha forestal.

En consecuencia, la forma reproductiva más segura es la modalidad vegetativa. Tales riesgos, junto a una restringida distribución areal hasta ahora, ameritan exploraciones y la búsqueda de nuevos sitios con $A$. bijuga, así como iniciar un programa de propagación y recuperación in situ $\mathrm{y}$, finalmente, definir una categoría de estatus de conservación de la cual hasta hoy carece. De acuerdo a la información de terreno hasta ahora disponible, la especie presenta una pequeña población en una única localidad, la extensión de la presencia estimada es menor a $10 \mathrm{~km}^{2}$ y existe una escasa cantidad de individuos maduros de la especie. Dado lo anterior y de acuerdo a las Categorías y Criterios de la Lista Roja de la UICN, versión 3.1 (2001), se sugiere para la especie la categoría de conservación provisoria de en Peligro Crítico (CR B1ab (v) +2ab (v); C2a (ii); D).

\section{AGRADECIMIENTOS}

Los autores agradecen a la Dirección de Investigación (DI) de la Universidad de Talca por el financiamiento del Proyecto VAF 600461 como también al Dr. Patricio Peñailillo del Instituto de Biología Vegetal y Biotecnología y a Luís Reyes Albornoz del Jardín Botánico de la Universidad de Talca.

\section{BIBLIOGRAFIA}

Amigo, J., J. San Martín \& L. Quintanilla. 2000. Estudio fitosociológico de los bosques de Nothofagus glauca (Phil.) Krasser del Centro-Sur de Chile. Phytoceonologie 30(2): 193-221.

Armesto, J., R. Rozzi, C. Smith-Ramírez, \& M. Arroyo. 1998. Conservation targets in South American temperate forests. Science 282: 1271-1272.

Arroyo, M.T.K., O. Matthei, M. MuÑoz-Schick, J. Armesto, P. Pliscoff, F. Pérez \& C. Marticorena. 2005. Flora de cuatro Reservas Nacionales en la Cordillera de la Costa de la VII Región (35$36^{\circ} \mathrm{S}$ ), Chile, y su papel en la protección de la biodiversidad regional. En: C. Smith-Ramírez, J. Armesto \& C. Valdovinos (eds.), Historia, biodiversidad y ecología de los bosques costeros de Chile, pp. 225-244. Editorial Universitaria, Santiago.

Braun-Blanquet, J. 1979. Fitosociología, bases para el estudio de comunidades vegetales. Blume Ediciones, Madrid. 820 pp.
BurKart, A. 1967. Sinopsis del género sudamericano de Leguminosas Adesmia DC. Darwiniana 14(2-3): 463-568.

Bustamante, R. \& J. Simonetti. 2005. Is Pinus radiata invading the native vegetation in Central Chile? Demographic responses in a fragmented forest. Biological Invasions 7: 243-249.

Bustamante, R., J. Simonetti, A. Grez \& J. San Martín. 2005. Fragmentación y dinámica de regeneración del bosque maulino: diagnóstico actual y perspectivas futuras. En: C. Smith-Ramírez, J. Armesto \& C. Valdovinos (eds.), Historia, diversidad y ecología de los bosques costeros de Chile, pp. 555-564. Editorial Universitaria, Santiago.

CAIN, S. 1950. Life forms and phytoclimate. The Botanical Review 16 (1): 1-32.

DierschKe, R. 1994. Pflanzensoziologie Grundlagen und Methoden. E. Ulmer, Stuttgart. 683 pp.

Donoso, C. 1975. Distribución ecológica de las especies de Nothofagus en la zona mesomórfica. Facultad de Ciencias Forestales, Universidad de Chile. Boletín Técnico 33: 1-21.

Donoso, C. 1993. Bosques templados de Chile y Argentina. Variación, Estructura y Dinámica. Editorial Universitaria, Santiago. 484 pp.

Donoso, C. 1996. Ecology of Nothofagus forest in Central Chile. In: Th. Veblen, R. Hill \& J. Read (eds.), The ecology and biogeography of Nothofagus forests, pp. 271-292. Yale University, USA.

Echeverría, C., D. Coomes, J. Salas, J. Rey-Benayas, A. Lara \& A. Newton. 2006. Rapid deforestation and fragmentation of chilean temperate forest. Biological Conservation 130: 481-494.

EllenberG, H. \& D. Mueller-Dombois. 1966. A Key to Raunkier plant life forms with revised subdivisions. Berichte des Geobotanischen Institutes der Eidgenoessischen Technischen Hochschule Stiftung Rübel 37: 56-73.

Fuentes, E. 1989. Ecología: introducción a la teoría de poblaciones y comunidades. Ediciones Universidad Católica de Chile, Santiago. 281 pp.

Fuenzalida, H. 1965. Biogeografía. En: Geografía Económica de Chile, Texto Refundido, CORFO, Santiago. pp. 228-267.

Gajardo, R. 1983. Sistema básico de clasificación de la vegetación nativa de Chile. Departamento de Silvicultura y Manejo, Facultad de Ciencias Agrarias, Veterinarias y Forestales, Universidad de Chile, Corporación Nacional Forestal, Santiago. $314 \mathrm{pp}$.

Gajardo, R. 1994. La vegetación natural de Chile. Clasificación y distribución geográfica. Editorial Universitaria, Santiago. 165 pp.

Grime, J. 1989. Estrategias de adaptación de las plantas y procesos que controlan la vegetación. Editorial Limusa Noriega Editores, México. 291 pp.

Guerrero, P. \& R. Bustamante. 2007. Can native tree species regenerate in Pinus radiata plantation in Chile? Evidence from field and laboratory 
experiments. Forest Ecology and Management 253: $97-102$

HaHn, S. \& P. Gómez. 2008. Hallazgo de Adesmia bijuga Phil. (Fabaceae) en la zona costera, Región del Maule, Chile central. Gayana Botánica 65 (1):119-121.

Hauenstein, E., C. Ramírez, M. Latsague \& D. Contreras. 1988. Origen fitogeográfico y espectro biológico como medida del grado de intervención antrópica en comunidades vegetales. Medio Ambiente 9(1): 140-142.

Izco, J. \& M. DEL Arco. 2003. Código internacional de nomenclatura fitosociológica. Universidad de La Laguna y Universidad de Santiago de Compostela Producciones Gráficas S.L., Galicia, España. 154 pp.

Kimmins, J. 1987. Forest Ecology. MacMillan Publishing Company \& Colier MacMillan Publishers. $531 \mathrm{pp}$.

KNAPP, C. 1984. Considerations on qualitative parameters and qualitative attributes in vegetation analysis and phytosociological relevés. In: R. Knapp (ed.), Sampling methods and taxon analysis in vegetation science. pp. 77-119. Dr. W. Junk Publication, La Haya.

Litton, C. \& R. Santelices. 1996. Comparación de las comunidades vegetales en bosques de Nothofagus glauca (Phil.) Krasser en la Séptima Región de Chile. Bosque 17(2): 77-86.

Mardones, M. 1995. La Cordillera de la Costa: caracterización físico-ambiental y regiones morfoestructurales. En: C. Smith-Ramírez, J. Armesto \& C. Valdovinos (eds.), Historia, biodiversidad y ecología de los bosques costeros de Chile, pp. 39-59. Editorial Universitaria, Santiago.

Mateucci, S. \& A. Colma. 1982. Metodología para el estudio de la vegetación. OEA. Estado de Falcón, Venezuela. $168 \mathrm{pp}$.

Matthei, O. 1995. Manual de malezas que crecen en Chile. Alfabeta Impresores, Santiago, Chile. $543 \mathrm{pp}$.

Montenegro, G., G. Avila \& P. Schatte. 1983. Presence and development of lignotubers in shrubs of the Chilean matorral. Canadian Journal of Botany 61: 1804-1808.

Mueller-Dombois, D. \& H. Ellenberg. 1974. Aims and methods of vegetation ecology. John Wiley \& Sons, New York. 547 pp.

Muñoz, A. \& R. MurúA. 1989. Efecto de la reforestación con Pinus radiata sobre la diversidad y abundancia de los micromamíferos en un agrosistema forestal de Chile Central. Turrialba 39(2): 143-150.

Myers, N., R. Mittermeier, G. Fonseca \& J. Kent. 2000. Biodiversity hotspots for conservation priorities. Nature 403: 853-858.

Niemi, G. \& M. McDonald. 2004. Application of ecological indicators. Annual Review of Ecology, Evolution, and Systematics 35: 89-111.

Pauchard A., M. Aguayo \& P. Alaback. 2006.
Cuantificando la fragmentación del paisaje: las métricas y sus significados ecológicos. En: A. Grez, J. Simonetti \& R. Bustamante (eds.), Biodiversidad en Ambientes Fragmentados de Chile: patrones y procesos a diferentes escalas, pp. 41-67. Editorial Universitaria. Santiago, Chile.

PhilipPI, R.A. 1884. Descripción de algunas plantas nuevas chilenas. Anales de la Universidad de Chile 65: 57-65.

Pinochet, F. 1991. Los suelos forestales de la Región del Maule. Universum 6: 37-46. Editorial Universidad de Talca, Talca.

Ramírez, C. 1988. Formas de vida, fitoclimas y formaciones vegetales. El Arbol...Nuestro Amigo 4: 33-37.

Ramírez, C., F. Figueroa, R. Carrillo \& D. Contreras. 1984. Estudio fitosociológico de los estratos inferiores en un bosque de pino, Valdivia, Chile. Bosque (Valdivia) 5(2): 65-81.

Ramírez, C., C. San Martín \& M.L. Keim. 1997. Flora ruderal del antiguo basural de Ovejería (Osorno, Chile). Gayana Botánica 54(1): 19-30

Ramírez, C., C. San Martín \& P. OJeda. 1997. Muestreo y tabulación fitosociológica aplicados al estudio de los bosques nativos. Bosque 18(2): 19-27.

ReIche, C. 1897. Estudios críticos sobre la flora de Chile. Anales de la Universidad de Chile 97: 725-790.

Richardson, D.M. 1998. Ecology and Biogeography of Pinus. D.M. Richardson (ed.), Cambridge University Press, $527 \mathrm{pp}$.

Rodríguez, R., O. Matthei \& M. Quezada. 1983. Flora Arbórea de Chile. Editorial Universidad de Concepción, Chile. 408 pp.

Rundell, P. 1981. The matorral zone of Central Chile. In: F. di Castri, D.W. Goodall \& R.L. Specht (eds.), Mediterranean-type shrublands, pp. 175201. Elsevier, Amsterdam, Netherland.

Sala, E., F. Chapin, J. Armesto, E. Berlow, J. Bloomfield, R. Dirzo, E. Huber-Sanwald, L. Huenneke, R. Jackson, A. Kinzig, R. Leemans, D. Lodge, H. Mooney, M. Oesterheld, N. Poff, M. Sykes, B. Walker, M. Walter \& D. Wall. 2000. Global biodiversity scenarios for the year 2100. Science 287: 1770-1774.

SAN MARTín, J. 2005. Vegetación y diversidad florística en la Cordillera de la Costa de Chile Central (344ㄴ'-3550’S). En: C. Smith-Ramírez, J. Armesto \& C. Valdovinos (eds.), Historia, biodiversidad y ecología de los bosques costeros de Chile, pp. 178-196. Editorial Universitaria, Santiago.

SAn Martín, J. \& C. Donoso. 1995. Estructura florística e impacto antrópico en el bosque maulino de Chile. En: J. Armesto, C. Villagrán \& M.T.K. Arroyo (eds.), Ecología de los bosques nativos de Chile, pp. 153-168. Editorial Universitaria, Santiago.

San Martín, J., C. San Martín \& C. Ramírez. 2001. Descripción de la estructura florística y vegetacional del matorral asociado a la rivera del 
Gayana Bot. 66(2), 2009

río Curanilahue (Cauquenes, VII Región, Chile). Revista Geográfica de Chile Terra Australis 46: $7-26$.

San Martín, J., J. Solerviscens, C. Ramírez, C. Sanmartín \& M. Elgueta. 1992. Estudio fitosociológico de los bosques pantanosos de Mirtáceas de la Región del Maule, Chile. Ciencias Forestales 8(1-2): 3-18.

Smith-Ramírez, C. 2004. The chilean coastal range: a vanishing center of biodiversity and endemism in South American temperate rainforest. Biodiversity and Conservation 13: 373-393.

Stoll, A. 2007. Hemerobia: una medida para evaluar el estado de conservación de comunidades vegetales nativas, Chloris Chilensis, Año $10 \mathrm{~N}^{\circ}$ 2. URL: www.chlorischile.cl.

Stoll, A. 2008. Hemerobia, un ejemplo de cálculo: el estado de conservación del bosque de pitao y coigüe (Pitavio-Nothofagetum) en Quebrada Honda, Región del Maule (VII), Chile. Chloris Chilensis, Año 11-Nº1. URL: www.chlorischile.cl.

Sukope, H. 1969. Der Einfluss des Menschen auf die Vegetation. Vegetatio 17(1/6): 360-371.

U.I.C.N. 2001. Categorías y criterios de la lista roja de la
UICN: versión 3.1. Comisión de Supervivencia de Especies de la UICN. UICN, Gland, Suiza y Cambridge, Reino Unido. ii +33 pp.

Ulriksen, P., M. Parada \& P. Aceituno. 1979. Climatología. Perspectivas de desarrollo de los recursos de la VII Región. IREN-CORFO Santiago, $\mathrm{N}^{\circ} 25,69 \mathrm{pp}$.

Villagrán, C. \& C. Le-Quesne. 1996. El interés biogeográfico-histórico de Chile Central-Sur. ¿Por qué debemos conservar su biota? En: M. Muñoz, H. Núñez \& J. Yáñez (eds.), Libro rojo de los sitios prioritarios para la conservación de la diversidad biológica en Chile, pp. 160-172. Corporación Nacional Forestal, Ministerio de Agricultura, Santiago.

Wikum, D. \& G. Shanholtzer. 1978. Application of the Braun-Blanquet cover-abundance scale for vegetation analysis in land development studies.Environmental Management 2(4): 323-329.

Zulohga, F., O. Morrone \& M. Belgrano. 2008. Catálogo de las Plantas Vasculares del Cono Sur (Argentina, Sur de Brasil, Chile, Paraguay, Uruguay). URL: www.darwin.edu.ar 